\title{
Detection of $\mathrm{O}_{2}$ Produced Abiotically on Habitable but Lifeless Planets around M-dwarfs
}

\author{
Tong Li and Feng Tian \\ Ministry of Education Key Laboratory for Earth System Modeling, Center for Earth System \\ Science, Tsinghua University, Beijing, China 100084
}

\begin{abstract}
High atmospheric abundances of oxygen has been widely considered to be a reliable biosignature for life on exoplanets in the habitable zones of all types of stars. Recently it was proposed that the unique UV spectra of observed planet-hosting M dwarfs could lead to the buildup of molecular oxygen in the atmospheres of habitable but lifeless planets around these stars (Tian et al. 2014). However, the detectability of the accumulated $\mathrm{O}_{2}$ was not modeled. In this work we developed a new line by line radiative transfer model based on HITRAN database and used the model to simulate the reflectivity in the visible and near IR range. We show that abiotically produced and maintained $\mathrm{O}_{2}$ in the $0.2 \%$ level is observable at $13105 \mathrm{~cm}^{-1}(0.76 \mu$ m) with the spectra resolution of 70 .
\end{abstract}

Keywords. exoplanets: biosignatures; detection: biosignatures; Habitability

\section{Introduction}

Many previous papers have been published regarding atmospheric oxygen as a biosignature. The first efforts including Earth-like volcanic outgassing predicts extremely low $\mathrm{O}_{2}$ mixing ratios, in the range of $10^{-15}$ bar at the surface (Walker 1977, Kasting et al. 1979) and a $10^{-5}$ mixing ratio above $40 \mathrm{~km}$ altitude (Kasting et al. 1979). For planets without volcanic activity but with Earth-like hydrological activities, models including rainout of oxidized and reduced species, which are produced through photochemistry, show that the $\mathrm{O}_{2}$ mixing ratio should remain below $10^{-11}$ bar at the surface (Kasting et al. 1984, Kasting 1990, 1993).

Rosenqvist and Chassefiere (1995) used a photochemical model to predict that the atmospheric $\mathrm{O}_{2}$ partial pressure could not exceed 5 mbar in an atmosphere with a $>95 \%$ $\mathrm{CO}_{2}$ mixing ratio, surface pressure between $1 \mathrm{mbar}$ and $10 \mathrm{bars}$, and a range of temperature and water vapor profiles. Kasting (1995) pointed out that Rosenqvist and Chassefiere (1995) neglected oxidation of the surface and volcanic outgassing of reducing gases and thus overestimated the atmospheric oxygen levels. For a planet closer to its star than the inner edge of the habitable zone, $\mathrm{H}_{2} \mathrm{O}$ should be lost to space rapidly in the form of hydrogen, and the accumulation of atmospheric oxygen could occur if oxygen escapes slower than half the hydrogen escape rate (Kasting 1997). But such an oxygen-rich atmosphere should be transient because oxygen reacts with reducing volcanic gases and the planet's surface (Kasting 1995).

With a more detailed photochemical model, Selsis et al. (2002) revisited the issue carefully. Their main findings are:

1) In the present Mars case $\left(\mathrm{CO}_{2}\right.$-dominant low pressure atmosphere with $3.18 .4 \mu \mathrm{m}$ precipitable water), an $\mathrm{O}_{2}$ column density on the order of $10^{20} \mathrm{~cm}^{-2}$ and an $\mathrm{O}_{3}$ column density on the order of $10^{15}$ to $10^{16} \mathrm{~cm}^{-2}$ are feasible. These oxygen contents cannot be observed by the proposed Darwin mission (Léger et al. 1996). 
2) In the early Mars case ( $\mathrm{CO}_{2}$-dominant 1-bar atmosphere and $1 \%$ humidity), $\mathrm{O}_{2}$ column density on the order of $10^{23} \mathrm{~cm}^{-2}$ and an $\mathrm{O}_{3}$ column density on the order of $10^{19}$ $\mathrm{cm}^{-2}$ can be reached. The high $\mathrm{O}_{2}$ content is detectable and therefore is not by itself considered a reliable biosignature in the early Mars case. Although the $\mathrm{O}_{3}$ content is also high, its IR feature is completely masked by the high $\mathrm{CO}_{2}$ content. And the detection of high $\mathrm{CO}_{2}$ content could provide a clue for possible abiotic $\mathrm{O}_{2}$ and $\mathrm{O}_{3}$ production in this case. Thus the detection of $\mathrm{O}_{3}$ in combination with $\mathrm{H}_{2} \mathrm{O}$ and $\mathrm{CO}_{2}$ is proposed to be a reliable biosignature.

3) In the case of a humid $\mathrm{CO}_{2}$-dominant dense atmosphere $\left(3.2\right.$ bar $\mathrm{CO}_{2}, 0.8$ bar $\mathrm{N}_{2}$, $100 \%$ surface humidity), a $0.3 \% \mathrm{O}_{2}$ mixing ratio and a $10^{-7} \mathrm{O}_{3}$ mixing ratio can be obtained at the surface. In a humid $\mathrm{N}_{2}$-dominant less dense atmosphere $\left(0.2\right.$ bar $\mathrm{CO}_{2}$, 0.8 bar $\mathrm{N}_{2}$ ), the calculated oxygen contents are significantly lower. In both cases the IR features of $\mathrm{O}_{3}$ cannot be detected because of the low $\mathrm{O}_{3}$ concentration and/or the high $\mathrm{CO}_{2}$ content.

Selsis et al. (2002) also pointed out that a planet with permanent supply of water and $1 \%$ atmospheric content of $\mathrm{O}_{2}$ does not produce enough $\mathrm{O}_{3}$ to produce a "false positive" $\mathrm{O}_{3}-\mathrm{H}_{2} \mathrm{O}-\mathrm{CO}_{2}$ biosginature. Thus Selsis et al. (2002) concluded that the combination of $\mathrm{O}_{3}, \mathrm{H}_{2} \mathrm{O}$, and $\mathrm{CO}_{2}$ is a robust biosignature for humid atmospheres, while $\mathrm{O}_{2}$ concentration is not.

Segura et al. (2007) agreed with Selsis et al. (2002) that high $\mathrm{O}_{2}$ buildup from $\mathrm{CO}_{2}$ photolysis is possible for planets with a weak hydrological cycle, either water-free or planets with a frozen surface. However, they argue that these hydrologically inactive planets can be identified by the nondetection of $\mathrm{H}_{2} \mathrm{O}$ in the visible and MIR spectra, and thus should not pose a true "false-positive" test for exoplanet life. For lifeless planets with Earth-like hydrological cycles and outgassing rates, Segura et al. (2007) argued that the accumulation of $\mathrm{O}_{2}$ and $\mathrm{O}_{3}$ are unlikely because of the rainout of both oxidizing and reducing species from the atmosphere. Most Recently $\mathrm{Hu}$ et al. (2012) found a buildup of oxygen in a 1-bar $\mathrm{CO}_{2}$ atmosphere with no outgassing of reducing gases.

Another series of papers studied the $\mathrm{O}_{3}$ concentration by fixing the atmospheric $\mathrm{O}_{2}$. Segura et al. (2003) found that the $\mathrm{O}_{3}$ column density in an atmosphere with $>0.2 \% \mathrm{O}_{2}$ should be in the order of $10^{17} \mathrm{~cm}^{-2}$ and that this level of $\mathrm{O}_{3}$ should be visible in the thermal IR. Segura et al. (2005) used the present Earth's $\mathrm{O}_{2}$ concentration to study the concentrations of $\mathrm{O}_{3}$ and other biogenic gases such as $\mathrm{CH}_{4}, \mathrm{CH}_{3} \mathrm{Cl}, \mathrm{N}_{2} \mathrm{O}$ and found that these gases could reach detectable levels for the UV flux levels of the active $\mathrm{M}$ dwarfs AD Leo and GJ643. Grenfell et al. (2013) used the present Earth's $\mathrm{O}_{2}$ concentration but with lower UVB fluxes from $M$ dwarfs to find a decrease in $\mathrm{O}_{3}$ number density at the surface of $\mathrm{M}$ dwarf planets.

A planet closer to its star than the inner edge of the habitable zone could lose its water rapidly by $\mathrm{H}_{2} \mathrm{O}$ photodissociation followed by hydrogen escape, leaving a large amount of oxygen in its atmosphere at least transiently (Kasting 1997). However, because such a planet is outside of the habitable zone, its oxygen-rich atmosphere does not constitute a true "false positive" biosignature.

However, no previous paper on atmospheric oxygen concentrations used realistic UV spectra of planet-hosting $\mathrm{M}$ dwarfs because such data were not available back then. France et al. (2013) is the first effort to observe the UV spectra of such stars with adequate spectral resolution and the observations show that planet-hosting $\mathrm{M}$ dwarfs have FUV/NUV ratios almost 100 times greater than that of our Sun. Tian et al. (2014) investigated photochemistry in the atmosphere of lifeless Earth-mass planets in the habitable zones of such stars and found that $0.2 \%$ of $\mathrm{O}_{2}$ can be accumulated in an atmosphere with $5 \% \mathrm{CO}_{2}$ in this condition. The $\mathrm{O}_{3}$ concentration in the photochemical model is also 
Table 1. spectral bands and resolutions in the model (follow Table 1 in DM02)

\begin{tabular}{lllllllllll}
\hline$v\left(\mathrm{~cm}^{-1}\right)$ & 5330 & 7110 & 7895 & 8815 & 10625 & 12175 & 13105 & 13815 & 14515 \\
\hline Species & $\mathrm{H}_{2} \mathrm{O}$ & $\mathrm{H}_{2} \mathrm{O}$ & $\mathrm{O}_{2}$ & $\mathrm{H}_{2} \mathrm{O}$ & $\mathrm{H}_{2} \mathrm{O}$ & $\mathrm{H}_{2} \mathrm{O}$ & $\mathrm{O}_{2}$ & $\mathrm{H}_{2} \mathrm{O}$ & $\mathrm{O}_{2}$ \\
\hline$v / \Delta v$ & 11 & 10 & 72 & 19 & 17 & 35 & 69 & 37 & 54 \\
\hline
\end{tabular}

high. The high oxygen concentration in the simulations is a result of the high FUV/NUV ratio. FUV is a source of oxygen from $\mathrm{CO}_{2}$ photolysis, while NUV is a source of $\mathrm{H}_{2} \mathrm{O}_{2}$ and $\mathrm{HO}_{2}$ species, which act as catalysts for the recycling of oxygen and $\mathrm{CO}$ back to $\mathrm{CO}_{2}$. With decreasing NUV flux, the recycling of oxygen to $\mathrm{CO}_{2}$ is slowed down which leads to the accumulation of oxygen in the atmosphere (Selsis et al. 2002, Tian et al. 2014).

Although Tian et al. (2014) showed oxygen accumulation, they did not carry out radiative transfer calculations to evaluate whether the oxygen in lifeless but habitable planets around M dwarfs can be observed. Results in Kaltenegger et al. (2007) for the Earth's atmospheric composition during different epochs in the evolution history are cited to support the conclusions in Tian et al. (2014). In this work we developed a new line by line radiative transfer model based on the HITRAN database. In the next section we describe and validate the model with present Earth's atmospheric composition, followed by simulations results of 3 cases: present Earth $\left(21 \% \mathrm{O}_{2}\right)$, abiotic habitable Earth-mass planet around solar-type stars, and abiotic habitable Earth-mass planet around stars with high FUV/NUV ratios. Section 3 contains the discussions and conclusions.

\section{Model Description, Validation and Results}

The HITRAN database (Rothman et al. 2009) is a well established source of basic data when building a model to calculate attenuation of light in a planetary atmosphere. In our line-by-line model (LT model), the absorption cross sections (in spectra resolution of $0.1 \mathrm{~cm}^{-1}$ ) of $\mathrm{O}_{2}$ and $\mathrm{H}_{2} \mathrm{O}$ under different atmospheric pressure and temperature values are calculated by considering Voigt profiles, the cutoff distance of which is set to 50 times the pressure broadening halfwidth or the Doppler halfwidth, whichever is greater. For present Earth we use the atmospheric profiles in the AFGL Atmospheric Constituent Profiles. For the abiotic Earth-mass planets under solar and M dwarf UV radiation we use the profiles calculated in Tian et al. (2014). The optical depths and transmission are calculated in each atmospheric layer. To compute reflectivity the light is transmitted twice through the atmosphere. The incident angle is assumed to be 60 degrees, similar to that in Des Marais et al. (2002, DM02 in the following).

To validate the LT model, we compared the model calculated reflectivity in Table 1 against Fig. 7 and 16 in DM02. Note that although $\mathrm{CO}_{2}$ features are not listed in Table 1, $\mathrm{CO}_{2}$ is included in the LT model. For frequency windows between the features listed in Table 1, a constant resolution $100 \mathrm{~cm}^{-1}$ is used.

Fig. 16 in DM02 shows reflectivity as functions of frequency for 4 different levels of $\mathrm{O}_{2}(0,1 \%, 21 \%$, and $50 \%)$. The DM02 reflectivity at 7895,13105 , and $14515 \mathrm{~cm}^{-1}$ are $0.8,0.5$, and 0.8 respectively in the $21 \% \mathrm{O}_{2}$ case. Fig. 1 in this work shows the calculated reflectivity as a function frequency in an atmosphere made of $79 \% \mathrm{~N} 2$ and $21 \% \mathrm{O}_{2}$. Comparisons of Fig. 1 in this work and Fig. 16 in DM02 show that all important absorption feature of $\mathrm{O}_{2}$ are computed correctly.

For $\mathrm{H}_{2} \mathrm{O}$, Fig. 9 in DM02 shows reflectivity as functions of frequency at $5 \mathrm{H}_{2} \mathrm{O}$ mixing ratio levels (0, $10 \mathrm{ppmv}, 100 \mathrm{ppmv}, 1000 \mathrm{ppmv}$, and 1\%). The reflectivity in $1 \% \mathrm{H}_{2} \mathrm{O}$ case at 5330, 7110, 8815, 10625, 12175, and $13815 \mathrm{~cm}-1$ are $0,0,0.05,0.05,0.55$, and 


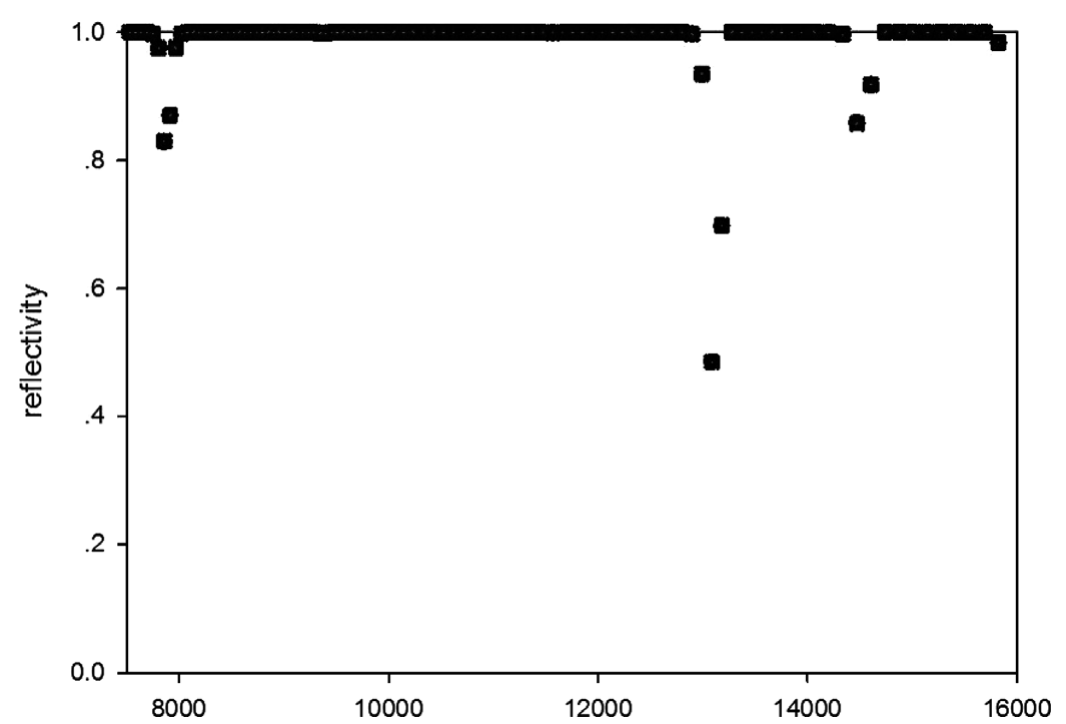

Figure 1. Reflectivity as a function of frequency considering an atmosphere made of $79 \% \mathrm{~N}_{2}$ and $21 \%$ of $\mathrm{O}_{2}$. The spectral features of $\mathrm{O}_{2}$ at 7895,13105 , and $14515 \mathrm{~cm}^{-1}$ are similar to those in Fig. 16 in Des Marais et al. (2002).

0.5 respectively. In comparison, Fig. 2 in this work shows that the reflectivity at these frequencies are $0,0,0.07,0.1,0.55$, and 0.5 respectively. Here the agreement between the LT model and the DM02 model is not perfect. But because we are interested in understanding whether $0.1 \%$ level of $\mathrm{O}_{2}$ can be detectable, the comparison between reflectivity between an atmosphere with this amount of oxygen and that with little oxygen is the key issue. Thus as long as the two cases have identical $\mathrm{H}_{2} \mathrm{O}$ content, the LT model can be used.

The atmospheric profiles used to estimate the detectability of $\mathrm{O}_{2}$ are shown in Fig. 3 . The data are from Tian et al. (2014) in which $\mathrm{H}_{2} \mathrm{O}$ content is controlled by the temperature profiles, which are identical in the solar UV case and in the M dwarf UV case.

Fig. 4 shows the reflectivity as functions of frequency in the present Earth case $(21 \%$ $\mathrm{O}_{2}$, black x symbols), the solar UV radiation case (zero $\mathrm{O}_{2}$, green circles), and the $\mathrm{M}$ dwarf UV radiation case $\left(0.2 \% \mathrm{O}_{2}\right.$, blue triangles). The signature of $0.2 \% \mathrm{O}_{2}$ can be clearly observed near $13105 \mathrm{~cm}^{-1}$. but not at near 12175 or $13815 \mathrm{~cm}^{-1}$ because the later two are dominated by the $\mathrm{H}_{2} \mathrm{O}$ absorption and the absorption caused by $0.2 \%$ level of $\mathrm{O}_{2}$ is overwhelmed.

A $0.2 \%$ surface level of $\mathrm{O}_{2}$ is indistinguishable from zero $\mathrm{O}_{2}$ at $14515 \mathrm{~cm}^{-1}$. This is although $\mathrm{H}_{2} \mathrm{O}$ absorption band is weak here (maximum cross section in the order of $5 \times 10^{-26} \mathrm{~cm}^{2}$ ), the $\mathrm{O}_{2}$ absorption in this frequency range is consisted of only a few narrow and strong lines (in the order of $5 \times 10^{-25} \mathrm{~cm}^{2}$ ). Thus a $0.2 \%$ level $\mathrm{O}_{2}$ cannot be detected with the low spectral resolution used here, consistent with the conclusion in Kaltenegger et al. (2007).

\section{Discussions and Conclusion}

In this work we developed a line-by-line radiative transfer model to study the detectability of $\mathrm{O}_{2}$ produced by atmospheric photochemistry driven by the unique UV radiation of observed planet-hosting $\mathrm{M}$ dwarfs. Our calculations confirmed that $0.2 \%$ 


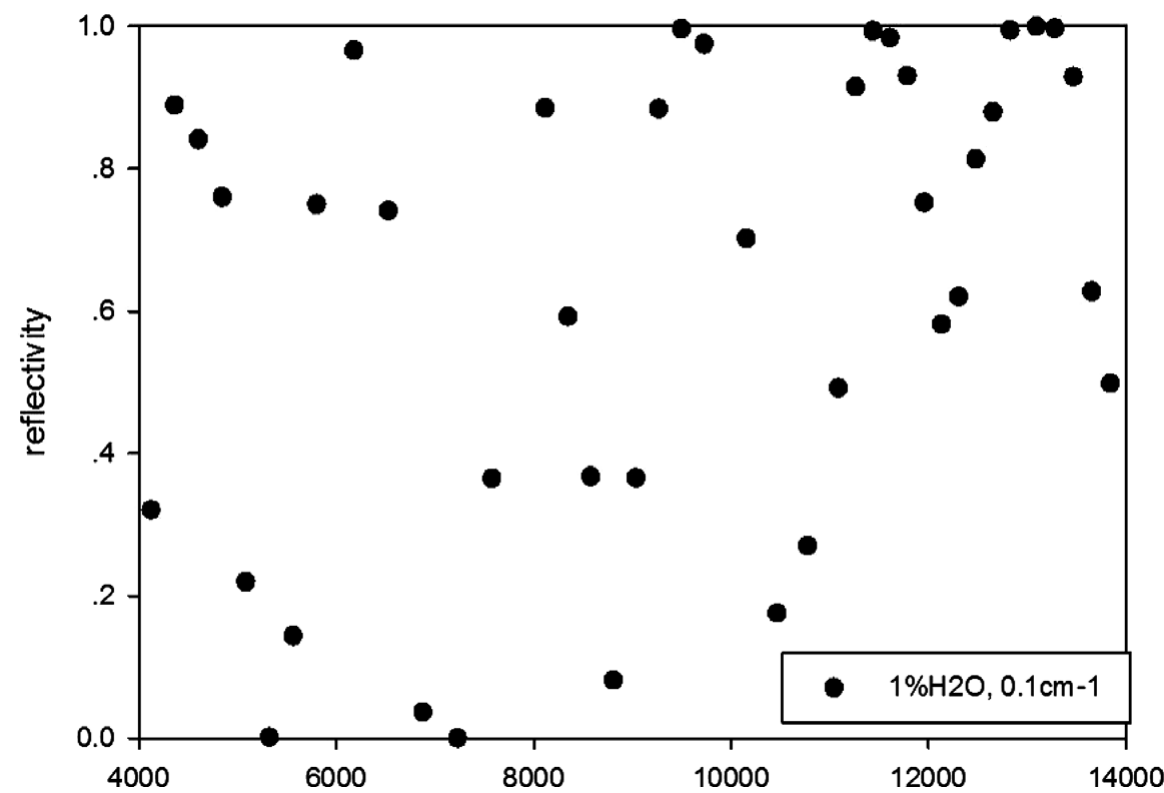

Figure 2. Reflectivity as a function of frequency considering an atmosphere made of $99 \% \mathrm{~N}_{2}$ and $1 \%$ of $\mathrm{H}_{2} \mathrm{O}$. The spectral features of $\mathrm{H}_{2} \mathrm{O}$ at $5330,7110,8815,10625,12175$, and $13815 \mathrm{~cm}^{-1}$ are similar to those in Fig. 7 in Des Marais et al. (2002).
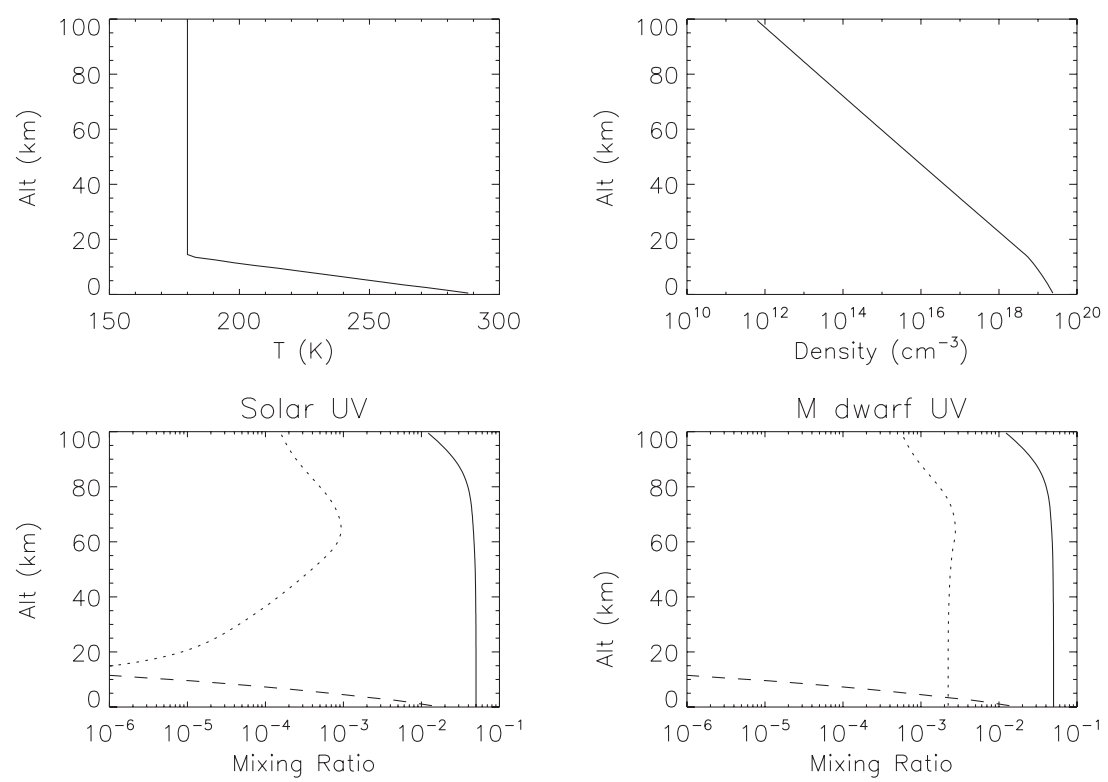

Figure 3. Atmospheric profiles in the solar UV and M dwarf UV cases based on Tian et al. (2014). Upper-left panel: temperature profile; upper-right panel: number density profile; lowerleft panel: profiles of $\mathrm{CO}_{2}$ (solid), $\mathrm{O}_{2}$ (dotted), and water vapor (dashed) in the solar UV case; lower-right panel: profiles of $\mathrm{CO}_{2}$ (solid), $\mathrm{O}_{2}$ (dotted), and water vapor (dashed) in the $\mathrm{M}$ dwarf UV case.

level of $\mathrm{O}_{2}$ in the atmosphere of a lifeless Earth-mass planet in the habitable zone of an $\mathrm{M}$ dwarf can be detected at $13815 \mathrm{~cm}^{-1}$. if the spectral resolution $\mathrm{R}$ (upsilon/ $\Delta v$ ) is 70 .

The model is preliminary in the sense that the effects of surface and clouds are not included into consideration. In this paper we focused on the visible frequencies and 


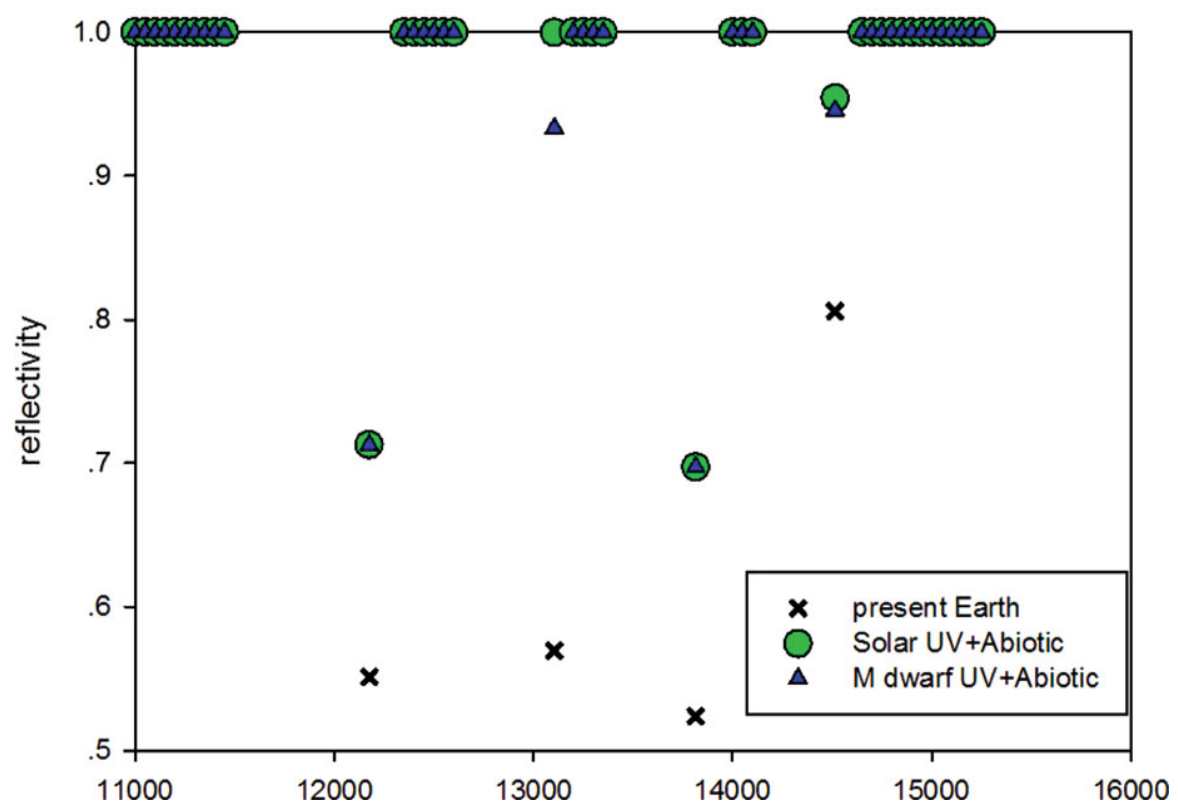

Figure 4. Reflectivity as functions of frequency in 3 cases. The black x symbols stand for the present Earth case $\left(21 \% \mathrm{O}_{2}\right)$. The green circles stand for lifeless planet under solar UV radiation case (close to $0 \% \mathrm{O}_{2}$ ). The blue triangles stand for lifeless planet under $\mathrm{M}$ dwarf $\mathrm{UV}$ radiation case $\left(0.2 \% \mathrm{O}_{2}\right.$ near the surface). The signature of $0.2 \% \mathrm{O}_{2}$ can be clearly observed near $13105 \mathrm{~cm}^{-1}$ but not at near 12175 or $13815 \mathrm{~cm}^{-1}$.

ignored the mid IR where $\mathrm{O}_{3}$ features are prominent. Thus although we are more certain that abiotically produced $\mathrm{O}_{2}$ are detectable, we are uncertain about the $\mathrm{O} 3$ detectability. These are important future research directions we will work on.

Another caveat is that the detection of $\mathrm{CO}_{2}$ is not included in this work. $\mathrm{CO}_{2}$ has strong spectral features in mid IR and in near IR, the later could be observed by the same mission designed to observe $\mathrm{O}_{2}$. This is important because if $\mathrm{CO}_{2}$ can be detected in near IR, one can potentially derive a relationship between atmospheric $\mathrm{O}_{2}$ and $\mathrm{CO}_{2}$ concentrations and compare it with the results in photochemical model. Such a comparison could be important for our effort to better understand the nature of oxygen in the target planet's atmosphere.

\section{Acknowledgement}

We thank helpful discussions with Dr. Renyu Hu and Dr. Xianglei Huang during the development of the LT model.

\section{References}

Des Marais, D. J. et al., Remote Sensing of Planetary Properties and Biosignatures on Extrasolar Terrestrial Planets. Astrobiology, 2, 153 (2002)

France, K. et al., The Ultraviolet Radiation Environment Around M dwarf Exoplanet Host Stars. ApJ, 763, 149 (2013)

Grenfell, J. L. et al., Potential Biosignatures in Super-Earth Atmospheres II. Photochemical Responses. Astrobiology, 13, 415 (2013)

$\mathrm{Hu}, \mathrm{R}$., et al., Photochemistry In Terrestrial Exoplanet Atmospheres. I. Photochemistry Model And Benchmark Case. ApJ, 761, 166 (2012) 
Kaltenegger, L., Traub, W. A., Jucks, K. W., Spectral Evolution Of An Earth-Like Planet. ApJ, $658,598(2007)$

Kasting, J. F. et al., Oxygen levels in the prebiological atmosphere. J. Geoph. Res., 84, 3097 (1979)

Kasting, J. F., Pollack, J. B. \& Crisp, D., Effects of high CO levels on surface temperature and atmospheric oxidation state of the early earth. J. Atmos. Chem., 1, 403 (1984)

Kasting, J. F., Bolide impacts and the oxidation state of carbon in the Earth's early atmosphere. Origins of Life, 20, 199 (1990)

Kasting, J. F., Early evolution of the atmosphere and ocean, in The Chemistry of Life's Origin (edited by J. M. Greenberg, C. X. Mendoza-Gomez and V. Pirronello). pp. 149-176. Kluwer. Dordrecht (1993)

Kasting, J. F., Earth's Early Atmosphere. Science, 259, 920 (1993)

Kasting, J. F., O2 concentrations in dense primitive atmospheres : commentary. Planetary and Space Sciences, 43, 11 (1995)

Kasting, J. F., Habitable zones around low mass stars and the search for extraterrestrial life. Origin of Life and Evolution of Biosphere, 27, 291 (1997)

Rosenqvist, J. \& E. Chassefiere, Inorganic chemistry of O2 in a dense primitive atmosphere. Planetary and Space Sciences, 43, 3 (1995)

Rothman, L. S. et al., The HITRAN 2008 molecular spectroscopic data base. Journal of Quantitative Spectroscopy \& Radiative Transfer, 110 (2009) 533

Segura, A. et al., Ozone Concentrations and Ultraviolet Fluxes on Earth-Like Planets around Other Stars. Astrobiology, 3, 689 (2003)

Segura, A. et al., Biosignatures from Earth-Like Planets Around M Dwarfs. Astrobiology, 5, 706 (2005)

Segura, A. et al., Abiotic formation of $\mathrm{O}_{2}$ and $\mathrm{O}_{3}$ in high- $\mathrm{CO}_{2}$ terrestrial atmospheres. $A \mathscr{E} A$, 472,665 (2007)

Selsis, F. et al., Signature of life on exoplanets: Can Darwin produce false positive detections? $A \& A, 388,985$ (2002)

Tian, F. et al., High Stellar FUV/NUV Ratio and Oxygen Contents in the Atmospheres of Potentially Habitable Planets. Earth and Planetary Science Letters, 22, 27 (2014)

Walker, J. C. G., Evolution of the Atmosphere (Macmillan, New York, 1977) 